\title{
Encuesta sobre las opiniones en torno a la ley de interrupción voluntaria del embarazo en profesionales de Salud Mental en Argentina
}

\author{
Juan Cristóbal Tenconi ${ }^{1}$, Maximiliano Cesoni ${ }^{2}$, \\ Mariana Lagos ${ }^{3}$, Viviana A. Peskin ${ }^{4}$
}

\begin{abstract}
1. Médico Psiquiatra y Psicoanalista. Coordinador de los cursos de médico especialista en psiquiatría del Instituto Superior de Formación de Postgrado, Asociación de Psiquiatras Argentinos (APSA). Docente adscripto, Departamento de Psiquiatría y Salud Mental, Facultad de Medicina, Universidad de Buenos Aires.

2. Médico Especialista en Psiquiatría. Ex Presidente del Capítulo de Psiquiatras en Formación, Asociación de Psiquiatras Argentinos (APSA). Hospital "José T. Borda", CABA.

3. Médica Psiquiatra (UBA). Presidente del Capítulo de Salud Mental, Derechos Humanos y Emergencias Sociales, Asociación de Psiquiatras Argentinos (APSA). Miembro del comité ejecutivo del Consejo Internacional de Rehabilitación de Víctimas de Tortura (IRCT).

4. Médica Especialista en Psiquiatría (UBA). Miembro de los Capítulos de Psiquiatras en Formación, Investigación en Psiquiatría y Práctica de la Docencia e Investigación Educativa en Salud Mental, Asociación de Psiquiatras Argentinos (APSA).
\end{abstract}

Autor correspondiente: Juan Cristóbal Tenconi, E-mail: juantenconi@gmail.com

\begin{abstract}
Resumen
El objetivo de esta investigación es conocer las opiniones de los profesionales de la Salud Mental en Argentina sobre la interrupción del embarazo en el contexto del debate parlamentario sobre esta temática. Con este fin, el equipo de investigación diseñó una encuesta que fue aplicada entre profesionales de la salud mental en el XXXIII Congreso Argentino de Psiquiatría de APSA. Resultados: El 91,8\% de los encuestados manifestaron estar informados sobre la actual discusión legislativa de la ley de interrupción voluntaria del embarazo. El 83,5\% de los profesionales que respondieron la encuesta está de acuerdo con la despenalización del aborto. Conclusiones: Dado que las consecuencias de un cambio de legislación tendrán un impacto en la salud de la población, nos parece que los resultados obtenidos deberían sumarse al debate actual.
\end{abstract}

Palabras clave: Interrupción legal del embarazo - Aborto - Leyes de salud.

SURVEY AMONG MENTAL HEALTH PROFESSIONALS IN ARGENTINA ON OPINIONS TOWARDS VOLUNTARY PREGNANCY INTERRUPTION

\begin{abstract}
The goal of this research is to learn the opinions and attitudes towards pregnancy interruption among Mental Health professionals in Argentina, taking into account the current Congress debate. In order to achieve the goal, the research team designed a questionnaire applied among mental health providers at the XXXIII Argentinian Psychiatry Congress, Asociación de Psiquiatras Argentinos (APSA). Results: $91.8 \%$ of the participants responded to be informed on current parliamentary discussion on voluntary pregnancy interruption (termination). 83.5\% of Mental Health providers who answered the survey agree to decriminalize abortion. Conclusions: Given that the consequences of changing the current legislation would have an impact in Public Health, we believe that these results should be taken into account in the current debate.
\end{abstract}

Key words: Legal termination of pregnancy - Abortion - Health laws. 


\section{Introducción}

El debate planteado en el Congreso de la Nación Argentina sobre la ley de interrupción voluntaria (1) del embarazo fue una expresión más del histórico reclamo por la legalización (2) del aborto planteado durante años por diversos actores de la sociedad civil en el país. Esta particular circunstancia motivó a dar al tema un espacio de visibilidad y reflexión en nuestro medio profesional (3) e impulsó la presente investigación.

La temática del aborto, sus consecuencias sanitarias y la legislación que lo habilita o sanciona constituyen un motivo de debate no solo en la Argentina sino también alrededor del mundo.

Según la Organización Mundial de la Salud (OMS), el estatus socioeconómico de las mujeres, en particular aquellas con menos recursos, es un importante factor a tener en cuenta en relación con las consecuencias del aborto para la salud, dado que son quienes presentan mayor riesgo de someterse a un aborto peligroso definido el mismo como aquellas prácticas realizadas sin personal de salud entrenado y sin acceso a los medios sanitarios requeridos. La probabilidad de que este procedimiento se realice en este grupo socioeconómico en embarazos avanzados es también mayor e incrementa el riesgo de defunciones y traumatismos (4). Un informe de las Naciones Unidas señala que aquellos países que tienen una legislación restrictiva en torno a la interrupción del embarazo presentan tasas de aborto no seguro cuatro veces más altas y tasas de mortalidad materna tres veces más altas que aquellos países con legislación menos restrictiva en torno a esta problemática (5).

Como plantea la Organización Mundial de la Salud (OMS) la poca disponibilidad de servicios, los costos elevados, la estigmatización, la objeción de conciencia del personal sanitario y los requisitos innecesarios, tales como períodos de espera obligatorios, modalidad del asesoramiento, junto con la legislación restrictiva, son obstáculos que incrementan el riesgo de la práctica del aborto para la salud y la vida de las mujeres.

Por otro lado, la OMS elaboró un Manual de práctica clínica para el aborto seguro (4)en el cual brinda informaciones a los profesionales de la salud en torno al tema. Señala, así, que cada profesional debe conocer la legislación nacional y regional, y plantea que se "deberían proteger y promoverla salud de las mujeres y las adolescentes y sus derechos humanos; la toma de decisiones informada y voluntaria; la autonomía en la toma de decisiones; la no discriminación; la confidencialidad y privacidad".

Históricamente según Marge Berer (6), la prohibición del aborto respondía principalmente a tres causas: el peligro que atravesaban las mujeres que buscaban realizarse un aborto, la consideración de esta práctica como un pecado o una inmoralidad, y/o la idea de que debía mantenerse la vida del feto antes que resguardar la vida y la autonomía de la mujer.

Revisando la situación de la legislación en torno al aborto a nivel mundial, en el 98\% de los países esta práctica está permitida para salvar la vida de la madre (6), en el $63 \%$ se permite el aborto vinculado a la salud materna y en el $62 \%$ para preservar la salud mental materna. El porcentaje de países desciende a menos del 50\% si se consideran como motivo para abortar los casos de violación o incesto (43\%) o las anomalías fetales (39\%). Sólo un $32 \%$ a nivel mundial permite el aborto debido a situaciones socio-económicas desfavorables y el 27\% teniendo como único requisito un pedido de la mujer (6).

Dentro de los países de la región, Uruguay, Cuba, Guyana Francesa y Puerto Rico tienen las legislaciones menos restrictivas en relación a la interrupción del embarazo. El Salvador, Honduras, Nicaragua y Haití, se encuentran en las antípodas, no permitiendo la interrupción voluntaria, sin contemplar ninguna excepción (4).

En América Latina (6), se ha visto, en los últimos años, distintas medidas e interpretaciones de las leyes favoreciendo menos restricción y mayor inclusión de causales para la interrupción voluntaria del embarazo. En los EE. UU. (7), que presenta una política menos restrictiva, varios estados están evaluando limitar la práctica que si bien no está legislada ha sido habilitada por un fallo de la Corte Suprema.

En la Argentina, el primer Código Penal, de 1886, penaliza el aborto en todas sus formas. Posteriormente se realizaron sucesivas reformas que fueron siendo más permisivas a la posibilidad de realizar un aborto no punible. En 1921 se introdujo un cambio retomado en 1984 (3, 8). En el año 2012 la Corte Suprema de Justicia de la Nación se expidió en el caso FAL (9) con un fallo que provee una interpretación concreta de la norma de 1921. Luego, en 2015, retomando ese fallo el Ministerio de Salud de la Nación estableció el Protocolo para la Atención Integral de las Personas con Derecho a la Interrupción Legal del Embarazo (10) que tiene como objetivo el acceso a esta práctica de acuerdo a la normativa vigente.

Según el Código Civil y Comercial, que plantea los derechos humanos como rectores de la interpretación, el aborto se permite si "el embarazo es producto de una violación, entendida como cualquier relación sexual no consentida o en un marco de violencia de género" y "Si el embarazo pone en riesgo la vida o la salud, definida en la legislación nacional e internacional como salud integral, es decir, en sus dimensiones biológica, psicológica y social (OMS/ Ley Básica de Salud)" (11).

Existen controversias en relación a las implicancias en la salud física y mental de aquellas mujeres que abor$\tan (20)$. Las consecuencias de esta práctica en la salud parecieran estar vinculadas al contexto de clandestinidad, y no al aborto en sí mismo. Un trabajo de 1992 (12) reportó que los estudios citados que denostaban el aborto tenían menor rigurosidad metodológica que los que planteaban una conducta más permisiva frente a este. Remarcaba que el problema era más legal y político que sanitario. Coleman, en una revisión de la literatura efectuada en 2011, plantea que el aborto conlleva problemas mentales a posteriori. Sin embargo, un reciente artículo publicado en JAMA (15), en 2019, señala que entre las mujeres que buscaron el aborto aquellas cuyo embarazo 
llegó a término, tuvieron peor salud física que aquellas que abortaron. Existen estudios que dan cuenta que un aborto en el contexto de un embarazo no deseado no implica mayor riesgo de desarrollar sintomatología psiquiátrica en comparación con embarazos a término en el mismo contexto (13). Diversos artículos ponen el foco en el hecho del embarazo no deseado, no tanto en la interrupción o no, y plantean estrategias desde la salud mental en el acompañamiento (14) y toma de decisiones de aquellas mujeres atravesando esa situación.

Desde los Capítulos de Salud Mental de la Mujer, Derechos Humanos y Emergencias Sociales de la Asociación de Psiquiatras Argentinos (APSA) se vienen realizando actividades cuyo objetivo ha sido, y es, comprender la complejidad de la problemática del aborto en el marco institucional. En especial los aspectos de la subjetividad y los efectos en la salud mental que la ilegalidad y los prejuicios sociales hacen pesar sobre esta práctica. El Congreso Argentino de Psiquiatría de APSA resultó un escenario propicio para explorar esta temática entre los profesionales de la Salud Mental.

Este trabajo intenta una exploración contextual sobre un tema directamente ligado a la práctica profesional de los psiquiatras y a la salud mental, particularmente de las mujeres $(13,15,16)$, el cual constituye un tema pendiente en la agenda de la Salud Pública.

En consecuencia, el objetivo de la presente investigación fue conocer las opiniones de los profesionales del campo de la Salud Mental asistentes al Congreso Argentino de Psiquiatría del año 2018 sobre la interrupción del embarazo en el contexto del debate parlamentario sobre esta temática que se propone en el Congreso Nacional.

\section{Materiales y métodos}

El equipo de investigación confeccionó una encuesta anónima y voluntaria para conocer las opiniones de los profesionales asistentes al XXXIII Congreso Argentino de Psiquiatría de APSA. Ella fue entregada al momento de la inscripción en formato papel a la mayoría de los asistentes del Congreso entre el 18 y el 21 de abril de 2018, y distribuida en algunos de los salones de actividades. Los encuestados devolvían la encuesta respondida al personal que realizaba las inscripciones. En caso de no poder realizarla en ese momento y lugar, la encuesta estaba disponible en un link para llenar el cuestionario en la misma ventana de tiempo.

La encuesta constaba de 9 preguntas (ver Anexo 1). Las primeras 4 sobre información sociodemográfica (edad, género, profesión, socio de APSA). Luego dos preguntas en relación al conocimiento de la legislación vigente actualmente sobre la interrupción del embarazo y la discusión parlamentaria en este tema; tres preguntas con opción múltiple en relación a la opinión del profesional sobre distintos aspectos del tema y seis afirmaciones tipo likert con cinco opciones de respuesta (muy de acuerdo, algo de acuerdo, algo en desacuerdo, muy en desacuerdo, no sé).

\section{Análisis estadístico}

El software utilizado para realizar el análisis estadístico fue SPSS Statistics 24. Aquellas variables dicotómicas se analizaron con chi cuadrado, mientras que las variables continuas con test t. Se consideró estadísticamente significativo un valor de $\mathrm{p}<0.05$. En algunos momentos del análisis de los resultados, y con el fin de simplificar la lectura, hemos agrupado las respuestas tipo likert en tres grupos: a) De acuerdo (donde incluimos muy de acuerdo y algo de acuerdo), en b) Desacuerdo (unificando donde incluimos muy en desacuerdo y algo en desacuerdo), c) No sé.

\section{Resultados}

En el momento de la acreditación al congreso 1006 asistentes sobre los 5600 inscriptos contestaron la encuesta, o sea cerca del 18\% (66,8\% mujeres, 659 ; y 33,2\% varones, 328).

En relación al conocimiento de la legislación vigente, el 79\% $(n=776)$ refirió que la interrupción del embarazo es legal actualmente frente a riesgo de salud/violación, un $13 \%(n=128)$ señala que en ninguna circunstancia es posible la interrupción.

El 30\% de aquellos que desconocían la legislación actual se encuentran entre los 20 y los 39 años, 41\% entre 40 y 59 años y el 27,4\% fueron mayores de 60 años. Dentro de aquellos que conocen la legislación, 37,4\% se encuentran entre 20 y 39 años, $43 \%$ entre 40 y 59 años y $18,5 \%$ en aquellos mayores de 60 años.

Tabla 1.

\begin{tabular}{l|c}
\hline Variables sociodemográficas & n (\%) \\
\hline Edad Media & $45,67(\mathrm{SD} 14,1)$ \\
\hline Género (contestaron 987) & $328(33,2)$ \\
\hline Masculino & $659(66,8)$ \\
\hline Femenino & \\
\hline Profesión (contestaron 991) & 403 \\
\hline Psiquiatra & 71 \\
\hline Médico no psiquiatra & 243 \\
\hline Lic. en Psicología & 24 \\
\hline Lic. en Trabajo Social & 112 \\
\hline Otros & \\
\hline Socios de APSA (contestaron 998) & $217(21,7)$ \\
\hline SI & $781(78,3)$ \\
\hline NO & \\
\hline
\end{tabular}

El 91,8\% (n=903) de los encuestados manifestaron estar informados sobre la actual discusión legislativa sobre ley de interrupción voluntaria del embarazo. El 83,5\% de los profesionales que respondieron la encuesta $(n=832)$ está de acuerdo con la despenalización del aborto. En la Tabla 2 se observan los resultados obtenidos de las preguntas tipo likert. 
Tabla 2. Resultados obtenidos de las preguntas tipo likert.

\begin{tabular}{|c|c|c|c|c|c|}
\hline & $\begin{array}{l}\text { Muy de } \\
\text { acuerdo }\end{array}$ & $\begin{array}{l}\text { Algo de } \\
\text { acuerdo }\end{array}$ & $\begin{array}{c}\text { Algo en } \\
\text { desacuerdo }\end{array}$ & $\begin{array}{c}\text { Muy en } \\
\text { desacuerdo }\end{array}$ & No sé \\
\hline Si la vida de una mujer corre peligro & $90 \%$ & $5 \%$ & $1 \%$ & $3 \%$ & $1 \%$ \\
\hline Si una mujer quedó embarazada debido a una violación & $82 \%$ & $11 \%$ & $1 \%$ & $5 \%$ & $1 \%$ \\
\hline Si el feto tiene una malformación incompatible con la vida extrauterina & $80 \%$ & $10 \%$ & $4 \%$ & $5 \%$ & $1 \%$ \\
\hline Si una mujer así lo decide & $59 \%$ & $13 \%$ & $9 \%$ & $17 \%$ & $2 \%$ \\
\hline Si la mujer y su familia carecen de recursos económicos & $46 \%$ & $14 \%$ & $12 \%$ & $25 \%$ & $3 \%$ \\
\hline Si una mujer quedó embarazada porque falló el método anticonceptivo & $52 \%$ & $12 \%$ & $9 \%$ & $23 \%$ & $4 \%$ \\
\hline
\end{tabular}

El 95,1\% de aquellos que contestaron la encuesta está de acuerdo con permitir la realización de un aborto en el caso en que la vida de la mujer corra peligro. El $92,4 \%$ está de acuerdo con legalizar el aborto en aquellos casos en que la mujer quedó embarazada debido a una violación. Vale recordar aquí que estas opciones coinciden con las causales ya vigentes en las que el aborto no es punible en Argentina. Dentro de los encuestados el 90,5\% está de acuerdo con legalizar el aborto si el feto tiene una malformación incompatible con la vida extrauterina, y un 70,4\% lo legalizaría si una mujer así lo decide. Esta última opción muestra un porcentaje menor de acuerdo entre los varones encuestados (30\% en género masculino, 70\% femenino). El promedio de edad de los que estuvieron de acuerdo con esta afirmación, fue de 44,9 años.

No hemos encontrado diferencias estadísticamente significativas en las respuestas dadas entre aquellos profesionales psiquiatras y aquellos profesionales de otras disciplinas. Tampoco entre nivel de acuerdo con respecto a cifras de legalización del aborto entre socios y no socios de nuestra institución. El 70\% de los entrevistados refirió estar a favor de la despenalización del aborto desde la semana 14 . Un 39\% está de acuerdo con que siempre sea posible, independientemente de las semanas de gestación.

\section{Discusión}

El debate planteado se sitúa en torno a la legalización y la despenalización del aborto, teniendo en cuenta que no son lo mismo. Se entiende por despenalizar eliminar una figura del Código Penal de la Nación. En cambio, legalizar implica, no sólo despenalizar, sino que el Estado debe generar las estructuras y herramientas para el acceso y realización segura de la práctica del aborto. Esto nos lleva a señalar, como primera cuestión que se desprende de los resultados obtenidos, que más de la mitad de los encuestados manifestó acuerdo con la interrupción del embarazo en función de la decisión de la mujer. Cabe destacar que la realización de la encuesta en el marco del Congreso Argentino de Psiquiatría coincidió con un contexto social en donde se debatía en el Congreso, en las calles y en la sociedad en su conjunto, la aprobación de una nueva ley al respecto. A pesar del rol de los profesionales de la Salud Mental y del impacto que la aprobación de la ley traería aparejado en la Salud Pública, es llamativo que sólo el 18\% de quienes recibieron la encuesta la respondieron. Esto podría estar vinculado con diversos factores: falta de interés por realizar una encuesta, sea la que fuese; escaso interés sobre el tema; percepción por parte de los profesionales de la Salud Mental que su opinión o su participación en una encuesta no tendría impacto o consecuencias en el debate actualmente planteado; factores relacionados con el contexto de la entrega (momento de la inscripción al Congreso); poca claridad en la entrega del cuestionario, etc. Es de remarcar que tanto senadores como diputados de la Nación han solicitado la opinión de expertos, convocando a hacer presentaciones con datos y argumentos en relación al nuevo proyecto de ley.

Teniendo en cuenta que las respuestas provienen de profesionales de la Salud Mental, y el momento histórico en el cual se realizó la encuesta, es importante remarcar que un 13\% desconoce la legislación actual vigente. Sin embargo sólo un 9\% refirió desconocer el debate legislativo actual sobre la temática. Existe controversia en relación a si el aborto tiene o no consecuencias en la salud mental de la mujer abortante. Las investigaciones científicas realizadas hasta la fecha dan cuenta de las dificultades existentes en la interpretación de los resultados. Como se dijo antes (vide supra) las mujeres que se someten a un aborto presentan repercusiones psíquicas que podrían explicarse por el contexto de clandestinidad y condena social y no por la interrupción en sí misma $(17,18)$.

Otras observaciones relevantes de la encuesta arrojan que los porcentajes varían de acuerdo a las causas que motivan la interrupción voluntaria del embarazo. En aquellas relacionadas con riesgos para la salud es similar el porcentaje entre hombres y mujeres, pero esta proporción cambia cuando se trata de una decisión de la mujer, en la que disminuye el acuerdo por parte de los varones (19). 


\section{Conclusiones}

Es de esperar que el Estado Argentino pueda tomar las decisiones de Salud Pública necesarias para responder a una demanda postergada en relación a la equidad, el cuidado de la salud y la salud mental de las mujeres. Estamos frente a una coyuntura que interpela en relación a la inclusión e igualdad de derechos, que sin duda representa el desafío de un cambio paradigmático que los profesionales de la salud mental tenemos por delante.

Es obvia la importancia de que los profesionales de Salud Mental se integren a los equipos interdisciplina- rios establecidos en las asesorías sobre interrupción del embarazo. A partir de los resultados de la encuesta se plantea el desafío de lograr una mayor implicación de los profesionales de Salud Mental a fin de contribuir a fortalecer las condiciones subjetivas y psíquicas de vulnerabilidad de la mujer que consulta.

\section{Agradecimientos}

Los autores agradecen a la Licenciada en Sociología Dalia Szulick por sus contribuciones para esta investigación.

\section{Anexo 1}

Estimados Colegas:

Este año se abrió el debate sobre la ley para la interrupción voluntaria del embarazo en el Congreso de la Nación en la que se discutirán diferentes proyectos que proponen modificar la legislación actual. Como trabajadores de la salud implicados en esta problemática nos interesa conocer la opinión de todos los participantes del Congreso XXXIII de Psiquiatría.

La encuesta es ANÓNIMA, solamente le llevará unos minutos responderla.

Marque con una $\mathrm{X}$ lo que considere correcto.

1. Edad: ............. años

2. Género:
a. Femenino
b. Masculino
c. Otro

3. Profesión:
a. Médico
Especificar especialidad :
b. Licenciado en psicología
c. Lic. en Trabajo social
d. Lic. en Musicoterapia
e. $\quad$ Otra
f. iEs socio de APSA?: si no

\section{De acuerdo a su conocimiento, la ley en nuestro país permite la interrupción del embarazo:}
a. En ninguna circunstancia
b. Sólo en circunstancias de riesgo de salud o de vida de la mujer y embarazo producto de violación
c. Siempre
d. No sé 
5. ¿Está informado sobre la actual discusión legislativa sobre la ley de interrupción voluntaria del embarazo?
a. Sí
b. No

6. ¿Cuál es su opinión respecto a la despenalización del aborto (en nuestro país se entiende por aborto a la interrupción del embarazo hasta la semana $\left.20^{\circ}\right)$ ?
a. Estoy de acuerdo
b. Estoy medianamente de acuerdo
c. No estoy de acuerdo
d. No sé

7. ¿Cuál es su nivel de acuerdo con la realización de un aborto bajo las siguientes circunstancias?

\begin{tabular}{|c|c|c|c|c|c|}
\hline & $\begin{array}{l}\text { Muy de } \\
\text { acuerdo }\end{array}$ & $\begin{array}{l}\text { Algo de } \\
\text { acuerdo }\end{array}$ & $\begin{array}{c}\text { Algo en } \\
\text { desacuerdo }\end{array}$ & $\begin{array}{c}\text { Muy en } \\
\text { desacuerdo }\end{array}$ & No sé \\
\hline \multicolumn{6}{|l|}{ Si la vida de una mujer corre peligro } \\
\hline \multicolumn{6}{|l|}{ Si una mujer quedó embarazada debido a una violación } \\
\hline \multicolumn{6}{|l|}{ Si el feto tiene una malformación incompatible con la vida extrauterina } \\
\hline \multicolumn{6}{|l|}{ Si una mujer así lo decide } \\
\hline \multicolumn{6}{|l|}{ Si la mujer y su familia carecen de recursos económicos } \\
\hline Si una mujer quedó embarazada porque falló el método anticonceptivo & & & & & \\
\hline
\end{tabular}

8. ¿Cree que debe ser legalizada la interrupción del embarazo (lo que implica el compromiso del gobierno a un acceso legal, seguro y gratuito)?
a. Siempre
b. Hasta la semana 24
c. Hasta la semana 14
d. Hasta la semana 8
e. Hasta la semana 6
f. Nunca
g. No sabe /no contesta

9. ¿Le interesaría participar de un debate sobre el tema en el ámbito de APSA?
a. No
b. $\quad \mathrm{Si}$
c. Posiblemente 


\section{Referencias bibliográficas}

1. Proyecto de Ley de Interrupción Voluntaria del Embarazo - Campaña Nacional por el Derecho al Aborto Legal Seguro y Gratuito. http://www.abortolegal.com.ar/proyecto-de-ley-presentado-por-la-campana/(2019)

2. Carta a senadores y senadoras exigiendo la aprobación del proyecto de Interrupción Voluntaria del Embarazo Campaña Nacional por el Derecho al Aborto Legal Seguro y Gratuito. http://www.abortolegal.com.ar/carta-a-senadores-y-senadoras-exigiendo-la-aprobacion-del-proyecto-de-interrupcion-voluntaria-del-embarazo/ (2019)

3. Historia del aborto en Argentina. Fundación Huésped https:// www.huesped.org.ar/informacion/derechos-sexuales-y-reproductivos/tus-derechos/interrupcion-legal-del-embarazo/ historia-del-aborto-en-argentina/ (2019).

4. Prevención del aborto peligroso. https://www.who.int/es/ news-room/fact-sheets/detail/preventing-unsafe-abortion (2019).

5. United Nations, Department of Economic and Social Affairs, Population Division (2014). Abortion Policies and Reproductive Health around the World (United Nations publication, Sales No. E.14.XIII.11).

6. Berer M. Abortion Law and Policy Around the World. Health Hum Rights. 2017,19: 13-27.

7. Zelikova NMB, Gretchen Goldman J. Abortion Bans Based on So-Called \&ldquo;Science\&rdquo; Are Fraudulent. Scientific American Blog Network https://blogs.scientificamerican.com/observations/abortion-bans-based-on-so-called-science-are-fraudulent/ (2019).

8. Attali L. Psychological aspects of abortion. J Gynecol Obstet Biol Reprod, 2016,45: 1552-1567.

9. https://www.cij.gov.ar/nota-8754-La-Corte-Suprema-preciso-el-alcance-del-aborto-no-punible-y-dijo-que-estos-casos-no-deben-ser-judicializados.html
10. http://www.msal.gob.ar/images/stories/bes/graficos/0000000875cnt-protocolo_ile_octubre\%202016.pdf

11. Código Penal de la Nación. http://servicios.infoleg.gob. ar/infolegInternet/anexos/15000-19999/16546/texact. htm\#15 (2019)

12. Wilmoth GH, Alteriis M de, Bussell D. Prevalence of Psychological Risks Following Legal Abortion in the U.S.A: Limits of the Evidence. Journal of Social Issues. 1982,48: 37-66.

13. https://www.aomrc.org.uk/wpcontent/uploads/2016/05/ Induced_Abortion_Mental_Health_1211.pdf

14. http://www.editorialpolemos.com.ar/docs/vertex/vertex117.pdf

15. Rubin, R. Among Women Seeking Abortion, Continuing Pregnancy Linked to Worse Physical Health. JAMA (2019) doi:10.1001/jama.2019.10313.

16. Ralph LJ, Schwarz EB, Grossman D, et al. Self-reported Physical Health of Women Who Did and Did Not Terminate Pregnancy After Seeking Abortion Services: A Cohort Study. Ann Intern Med. 2019;171:238-247. [Epub ahead of print 11 June 2019]. doi: https://doi.org/10.7326/M18-1666

17. Munk-Olsen, T., Laursen, T. M., Pedersen, C. B., Lidegaard, Ø. \& Mortensen, P. B. Induced First-Trimester Abortion and Risk of Mental Disorder. N Engl J Med, 2011, 364: 332-339.

18. https://www.apa.org/pi/women/programs/abortion/

19. Biggs MA, Upadhyay UD, McCulloch CE, Foster DG. Women's Mental Health and Well-being 5 Years After Receiving or Being Denied an Abortion: A Prospective, Longitudinal Cohort Study. JAMA Psychiatry. 2017, 74(2):169-178. doi:10.1001/jamapsychiatry.2016.3478

20. Reardon D. C. (2018). The abortion and mental health controversy: A comprehensive literature review of common ground agreements, disagreements, actionable recommendations, and research opportunities. SAGE open medicine, 6, 2050312118807624.doi:10.1177/2050312118807624. 